\title{
THEORETICAL FINE SPECTROSCOPY WITH SAC-CI METHOD: OUTER- AND INNER-VALENCE IONIZATION SPECTRA OF CO AND $\mathbf{N}_{2}$
}

\author{
Masahiro EHARA ${ }^{a 1}$, Mayumi IsHIDA $^{a}$ and Hiroshi NAKATSUJI ${ }^{a 2, b, *}$ \\ ${ }^{a}$ Department of Synthetic Chemistry and Biological Chemistry, Graduate School of Engineering, \\ Kyoto University, Katsura, Nishikyou-ku, Kyoto 615-8510, Japan; \\ e-mail: ${ }^{1}$ ehara@sbchem.kyoto-u.ac.jp, ${ }^{2}$ hiroshi@sbchem.kyoto-u.ac.jp \\ ${ }^{b}$ Fukui Institute for Fundamental Chemistry, Kyoto University, \\ 34-4 Takano Nishihiraki-cho, Sakyo-ku, Kyoto 606-8103, Japan
}

Received February 17, 2005 Accepted April 26, 2005

Dedicated to Professor Josef Paldus on the occasion of his 70th birthday.

Outer- and inner-valence ionization spectra of $\mathrm{CO}$ and $\mathrm{N}_{2}$ were studied by the SAC-Cl (symmetry-adapted-cluster configuration-interaction) general-R method. Fine details of experimental spectra of these molecules were reproduced and quantitative assignments of the peaks were proposed. Both outer- and inner-valence satellites were classified into the shakeup states including the valence or Rydberg excitations. For $\mathrm{CO}$, theoretical satellite spectrum up to $50 \mathrm{eV}$ was presented and the nine bands of 22-50 eV observed by EMS and eight bands of 22-34 eV by XPS (C-K) were characterized in detail. Numerous satellite peaks with distributed intensity were obtained and some of them, especially for bands 4, 5, 6 and 7, were predominantly described by triple-electron processes. For $\mathrm{N}_{2}$, the spectrum up to $45 \mathrm{eV}$ was calculated and the complex satellite peaks observed by XPS in the lower energy region 20-33 eV were interpreted. The detailed assignments for the $\left(2 \sigma_{\mathrm{g}}{ }^{-1}\right)$ satellite states in the higher-energy region 33-45 eV were also presented.

Keywords: SAC-CI; Valence ionization spectra; Satellite spectra; Ab initio calculations; Photoelectron spectroscopy; Quantum chemistry.

Many satellite peaks are usually involved in the inner-valence region of an ionization spectrum. They attracted considerable experimental and theoretical interests since they often provide information concerning electron correlations in molecules. Recently, extensive experimental studies of these peaks have been performed by high-resolution synchrotron radiation photoelectron spectroscopy (SRPES), X-ray photoelectron spectroscopy (XPS), and electron momentum spectroscopy (EMS). In parallel, advanced theoretical methods have also been used for detailed and quantitative assignments of these peaks. 
Out of the extensive work on the satellite peaks, the valence ionization spectra of $\mathrm{CO}$ and $\mathrm{N}_{2}$ molecules are of special interest, and have been studied in detail both experimentally ${ }^{1-14}$ and theoretically ${ }^{15-25}$. These spectra show many satellite peaks with complex band structures due to strong electron correlations. The satellite peaks continuously appear in both the outerand inner-valence regions. After the He I PES was reported for three main peaks by Turner et al. ${ }^{26}$, many careful and detailed studies on the innervalence satellite peaks have been performed by XPS ${ }^{1-4}, \operatorname{SRPES}^{5,6}, \mathrm{EMS}^{7,8}$, and He II PES ${ }^{9,10}$. French et al. ${ }^{8}$ observed the outer- and inner-valence spectra of $\mathrm{CO}$ up to $53 \mathrm{eV}$ by EMS and gave nine deconvoluted bands. Svensson et al. ${ }^{4}$ presented the detailed inner-valence spectra of $\mathrm{CO}$ and $\mathrm{N}_{2}$ by highresolution XPS. Recently, fine analysis was performed for the vibrational structure of some satellites of $\mathrm{CO}$ and $\mathrm{N}_{2}$ by high-resolution PES using synchrotron and He II radiations ${ }^{13}$ and ultraviolet photoelectron spectroscopy (UPS) ${ }^{14}$. Liu et al. ${ }^{12}$ also observed the fine structure in inner-valence PES of $\mathrm{CO}$ and $\mathrm{N}_{2}$ with a resolution better than $100 \mathrm{meV}$.

Theoretically, the outer- and inner-valence ionization spectra of these molecules have also been intensively studied by the configuration-iteration (CI) method ${ }^{15-20}$ and Green's function method ${ }^{20-23}$. Previously, we have also applied the symmetry-adapted-cluster $\mathrm{Cl}$ (SAC-CI) method to these spectra, however, the basis set and active space were limited since the study was aimed at comparison with the full-CI spectra ${ }^{24}$. Since the satellite peaks of these molecules are both numerous and complicated, the detailed characterization is still difficult and, actually, there are some controversial assignments. It is important to perform the accurate calculations using the sufficient basis sets, especially for the assignment of the high-lying shakeup states.

In this work, we studied the ionization spectra of $\mathrm{CO}$ and $\mathrm{N}_{2}$ in both the outer- and inner-valence regions by the SAC-CI general- $\mathrm{R}$ method with an extended basis set. The SAC/SAC-CI method ${ }^{27,28}$ has been successfully applied to molecular spectroscopic problems including ionization spectroscopy $^{24,29-37}$. In particular, the SAC-CI general-R method ${ }^{38,39}$ is designed to describe multielectron processes with high accuracy, and has been shown to be useful for studying the large number of states appearing in the ionization spectrum ${ }^{24,34-37}$. In the SAC-CI general-R method, not only singles and doubles but also triples, quadruples and higher-excitation operators are included as the linked excitation operators (R). Recently, we have succeeded in giving a fine analysis of the outer- and inner-valence ionization spectra of Group V and VI hydrides ${ }^{37}$. Details of the SAC-CI general-R method are given in ref. ${ }^{38}$ and in a review article recently summarized ${ }^{39}$. 


\section{COMPUTATIONAL DETAILS}

We studied vertical ionization spectra of $\mathrm{CO}$ and $\mathrm{N}_{2}$ and the experimental geometries ${ }^{40}$ were adopted; $r_{e}=1.12832 \AA$ and $r_{e}=1.09768 \AA$ for $C O$ and $\mathrm{N}_{2}$, respectively. We used valence triple-zeta (VTZ) basis sets of Ahlrichs ${ }^{41}$, augmented with two d-type polarization functions of $\zeta_{d}=1.097,0.318$ for C, $\zeta_{d}=2.314,0.645$ for $\mathrm{O}$, and $\zeta_{d}=1.654,0.469$ for $\mathrm{N}$. The exponents of the polarization functions of $\mathrm{C}, \mathrm{O}$ and $\mathrm{N}$ were taken from the correlationconsistent polarized VTZ (cc-pVTZ) sets ${ }^{42}$. In addition, s-, p- and d-type Rydberg functions were put on $\mathrm{C}, \mathrm{O}$ and $\mathrm{N}$ atoms: $\zeta_{\mathrm{s}}=0.023, \zeta_{\mathrm{p}}=0.021$, $\zeta_{d}=0.015$ for $C, \zeta_{s}=0.032, \zeta_{p}=0.028, \zeta_{d}=0.015$ for $O$, and $\zeta_{s}=0.028, \zeta_{p}=$ $0.025, \zeta_{d}=0.015$ for $\mathrm{N}$. The exponents of these Rydberg functions were due to ref. ${ }^{43}$. The resulting basis sets are $(12 s 8 p 5 d) /[8 s 5 p 3 d]$. The ionization spectra of $\mathrm{CO}$ and $\mathrm{N}_{2}$ were calculated by the SAC-Cl general-R method in both outer- and inner-valence regions up to around double-ionization threshold. Most of the shake-up states of these molecules were predominantly described by two-electron or three-electron processes, and therefore the R-operators were included up to quadruples. The active space consists of 5 occupied and 61 unoccupied MOs for these molecules; the 1s orbitals of $\mathrm{C}, \mathrm{O}$ and $\mathrm{N}$ were frozen as cores, and all the other MOs were included in the active space.

To reduce computational efforts, perturbation selection ${ }^{30}$ was carried out in the state-selection scheme. For the ground state, the threshold for the linked operator was $\lambda_{\mathrm{g}}=1 \times 10^{-7}$ a.u. and the unlinked terms were written as the products of the important linked terms whose SDCl coefficients were larger than 0.003 for $\mathrm{CO}$ and 0.001 for $\mathrm{N}_{2}$. For the ionized states, the threshold for the double excitation operators was $\lambda_{e}=1 \times 10^{-7}$ a.u., that for triples was $\lambda_{e}=1 \times 10^{-6}$ a.u., and that for quadruples was $\lambda_{e}=1 \times 10^{-5}$ a.u. for $\mathrm{CO}$ and $\lambda_{e}=1 \times 10^{-6}$ a.u. for $\mathrm{N}_{2}$. The thresholds of the $\mathrm{Cl}$ coefficients for calculating the unlinked operators in the SAC-CI method were 0.05 and $1 \times$ $10^{-8}$ for the $\mathrm{R}$ and $\mathrm{S}$ operators, respectively.

The ionization cross-sections were calculated using the monopole approximation 44,45 to estimate the relative intensities of the peaks. In calculating monopole intensity, the correlated SAC wave function was used for the ground state; namely both initial- and final- state correlation effects were included. In particular, the intensity of the ${ }^{2} \Delta,{ }^{2} \Delta_{\mathrm{g}}$ and ${ }^{2} \Delta_{\mathrm{u}}$ states arises only from the initial-state correlation effect.

Hartree-Fock (HF) SCF calculations were preformed using Gaussian $98^{46}$ and SAC/SAC-CI calculations were performed using the SAC-CI96 program system ${ }^{47}$. 


\section{IONIZATION SPECTRUM OF CO}

The outer- and inner-valence ionization spectra of CO up to $52 \mathrm{eV}$ were studied by the SAC-CI general-R method. The SAC-CI calculations were performed in $C_{2 v}$, therefore, the ${ }^{2} \Sigma^{+}$and ${ }^{2} \Delta$ states were calculated by ${ }^{2} A_{1}$ of $C_{2 v}$, while the ${ }^{2} \Pi$ states were obtained by ${ }^{2} B_{1}$ of $C_{2 v}$. To give the theoretical assignments in the energy region up to $52 \mathrm{eV}, 120$ and 30 ionized states were calculated for ${ }^{2} A_{1}$ and ${ }^{2} B_{1}$ symmetries of $C_{2 v}$, respectively. Note that the inner-valence satellite states with considerable intensities were associated with ${ }^{2} \Sigma^{+}$symmetry. The SAC-Cl general-R dimensions are summarized in Table I, together with those before the perturbation selection. Since most of the satellite states were predominantly described by two-electron process, the SD-Cl solutions were used for the reference states in the perturbation selection. The resulting dimensions were 113557 and 72637 for ${ }^{2} A_{1}$ and ${ }^{2} \mathrm{~B}_{1}$ symmetries, respectively.

TABLE I

The SAC-CI general-R dimensions for the ionized states of $\mathrm{CO}$ and $\mathrm{N}_{2}$

State

CO After perturbation selection

${ }^{2} \Sigma^{+},{ }^{2} \Delta$

${ }^{2} \Pi$

3

1

Before perturbation selection

${ }^{2} \Sigma^{+},{ }^{2} \Delta$

3

${ }^{2} \Pi$

1

$\mathrm{N}_{2} \quad$ After perturbation selection

${ }^{2} \Sigma_{\mathrm{g}}^{+},{ }^{2} \Delta_{\mathrm{g}}$
${ }^{2} \Pi_{\mathrm{g}}$
${ }^{2} \Sigma_{\mathrm{u}}^{+},{ }^{2} \Delta_{\mathrm{u}}$
${ }^{2} \Pi_{\mathrm{u}}$

2

0

1

1

Before perturbation selection
2

240

193

247

186
463

367

31245

24738

81846

47531

113557

72637

487

43662

2256746

2300898

41790

2246070

2288240

230

187

15637

160702

176571

12757

122963

135907

13688

119371

133293

12767

115915

128863

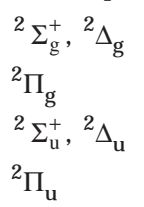

21854

1128294

1150390

20872

1123114

1144179

21808

1128452

1150508

20918

1122956

1144061 
The HF electronic configuration of $\mathrm{CO}$ is written as (core $)^{4}(3 \sigma)^{2}(4 \sigma)^{2}$ $(1 \pi)^{4}(5 \sigma)^{2}$. Figure 1 shows the ionization spectrum of $\mathrm{CO}$ in the outer- and inner-valence regions calculated by the SAC-CI general-R method in comparison with the SRPES spectrum ${ }^{6}$. In the theoretical spectrum, the calculated pole strengths were shown by solid lines and were convoluted using Gaussian envelopes with the full width half maximum (FWHM) 2.0 eV estimated by averaging FWHMs of three main peaks of the SRPES spectrum.
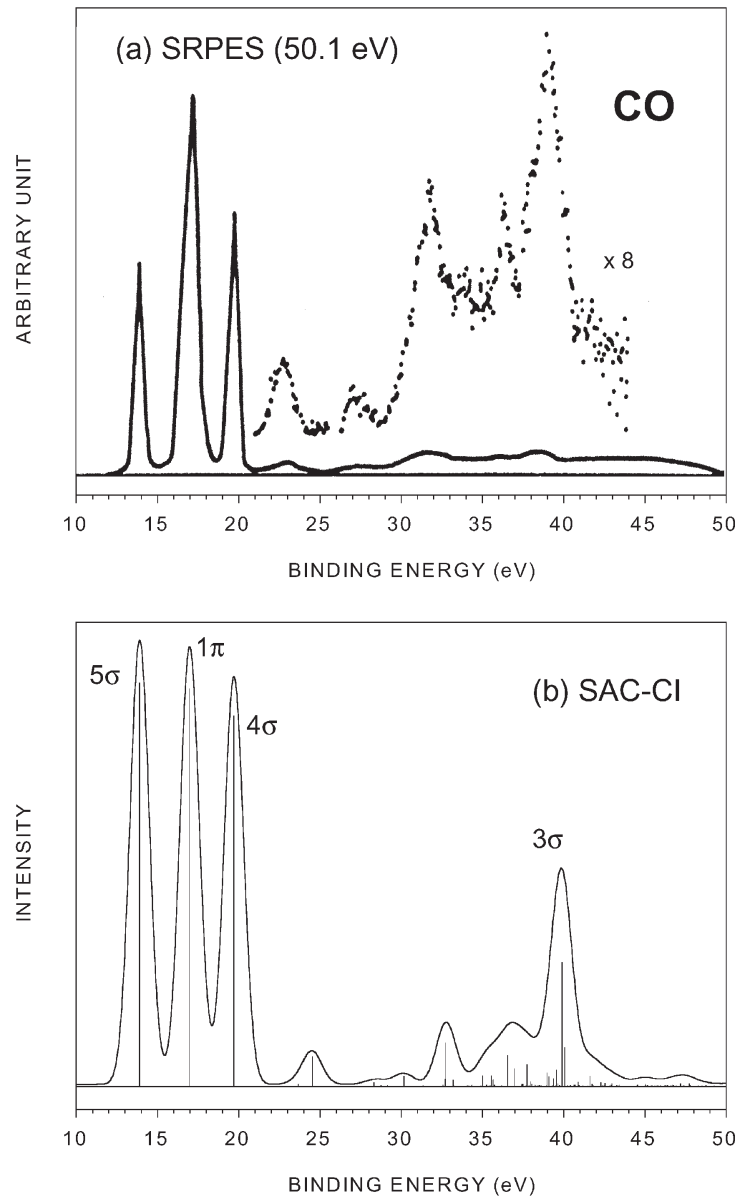

FIG. 1

The valence ionization spectra of CO by a SRPES $(50.1 \mathrm{eV})^{6}$, and b SAC-Cl general-R method. In the theoretical spectrum, the calculated pole strength of each peak is shown as a solid vertical line and is convoluted with a FWHM of $2.0 \mathrm{eV}$ 
The theoretical spectrum reproduces shape of the SRPES spectrum for both the main peaks and the satellites quite accurately. First, we discuss three main peaks: $\left(5 \sigma^{-1}\right),\left(1 \pi^{-1}\right)$ and $\left(4 \sigma^{-1}\right)$ states. Table II gives the results for these peaks with the experimental IPs by EMS ${ }^{8}, \mathrm{XPS}^{4}$ and He II PES ${ }^{9}$. The SAC-CI general-R results, $13.90,16.98$ and $19.70 \mathrm{eV}$, compare well with the experimental values 14.01, 17.03 and $19.6 \mathrm{eV}$ (EMS), 14.01, 17.0 and $19.7 \mathrm{eV}$ (XPS), and 14.01, 16.91 and $19.69 \mathrm{eV}$ (He II PES), for $\left(5 \sigma^{-1}\right),\left(1 \pi^{-1}\right)$ and $\left(4 \sigma^{-1}\right)$ states, respectively.

Next, the satellite peaks in the inner-valence region are discussed along with the EMS ${ }^{8}, \mathrm{XPS}^{4}$, and He II PES spectrum ${ }^{9}$. In ref. ${ }^{4}$, the assignments of the XPS spectrum were performed with the information from both deexcitation spectroscopy (DES) ${ }^{48}$ and UPS ${ }^{49}$ spectra, which gave a consistent characterization of the observed peaks. It was noted that the USP spectrum was useful since it emphasizes the ionization originating $\pi$ orbitals. In Fig. 2, the inner-valence satellite spectrum by the present calculation is compared with $\mathrm{EMS}^{8}$ and XPS ${ }^{4}$. In the EMS spectrum ${ }^{8}$, the sum of experimental binding energy spectra recorded at ten azimuthal angles $\phi=0-24^{\circ}$, was deconvoluted with Gaussian functions (2.5 eV FWHM) as shown by the dashed lines. Our theoretical spectrum was also convoluted with the equal line width of the EMS spectrum. Table II summarizes the IPS, monopole intensities and main configurations of the satellite peaks, whose IPs are up to $52 \mathrm{eV}$ with intensities greater than 0.002. Unfortunately, no experimental information on the intensity has been reported for comparison.

Nine satellite bands numbered $1-9$ were observed by EMS $^{8}$ in the energy region 22-52 eV, and eight peaks (C-K) of 22-34 eV were characterized in detail. As the lowest band, the medium peak at $24.1 \mathrm{eV}$ (band 1) was measured by EMS ${ }^{8}$. XPS ${ }^{4}$ and $\mathrm{He} \mathrm{II} \mathrm{PES}^{9}$ works also reported the peaks at 23.7 (C) and $23.4 \mathrm{eV}$, respectively. We assigned a ${ }^{2} \Sigma^{+}$state calculated at $24.53 \mathrm{eV}$ with the intensity 0.064 to this band. This state corresponds to the $\left(4 \sigma^{-1}\right)$ outer-valence satellite and is described by two-electron processes such as $\left(5 \sigma^{-1} 1 \pi^{-1} 6 \pi\right)$, which involves the valence-excitation to $\pi^{*}$ orbital. Contributions of the triples were also found and therefore the inclusion of triple and quadruple R-operators was important for describing this state. In the energy region below band 1 , He II PES and DES 48 observed a weak peak at $22.7 \mathrm{eV}$ (D). To the peak $D$, we assigned a ${ }^{2} \Pi$ state calculated at $23.65 \mathrm{eV}$ with the intensity 0.005 , which was mainly characterized as $\left(5 \sigma^{-2} 6 \pi\right)$. Next, the XPS and He II PES have also observed a very weak peak at $25.2 \mathrm{eV}$ (E) and $25.3 \mathrm{eV}$, respectively, in the shoulder of band 1 . The assignment of this state is still not definitive. Our method calculated no peak of ${ }^{2} \Sigma^{+}$or ${ }^{2} \Pi$ symmetries in the energy region $25-28 \mathrm{eV}$, but gave a ${ }^{2} \Delta$ state at $26.12 \mathrm{eV}$ with 


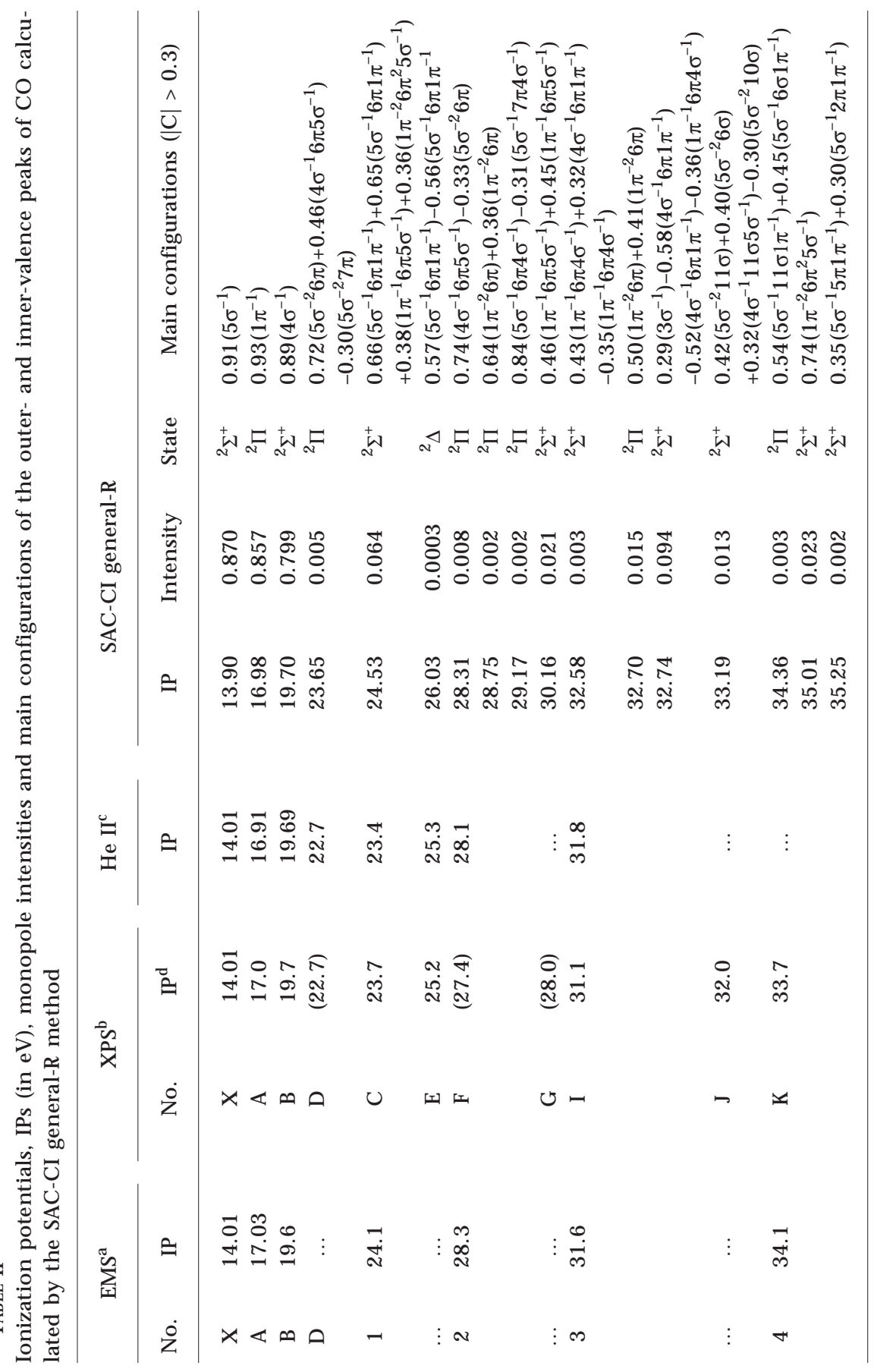




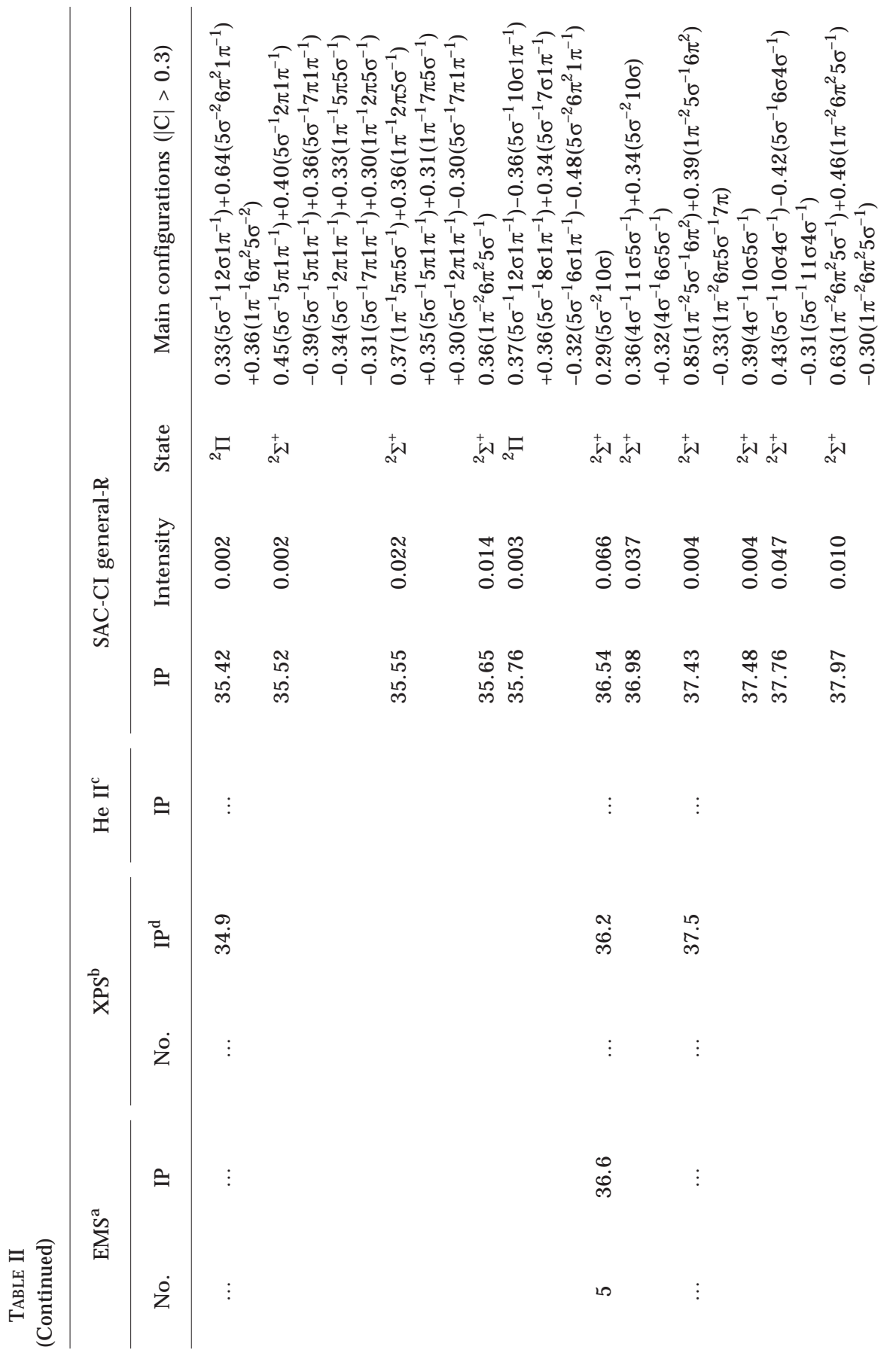




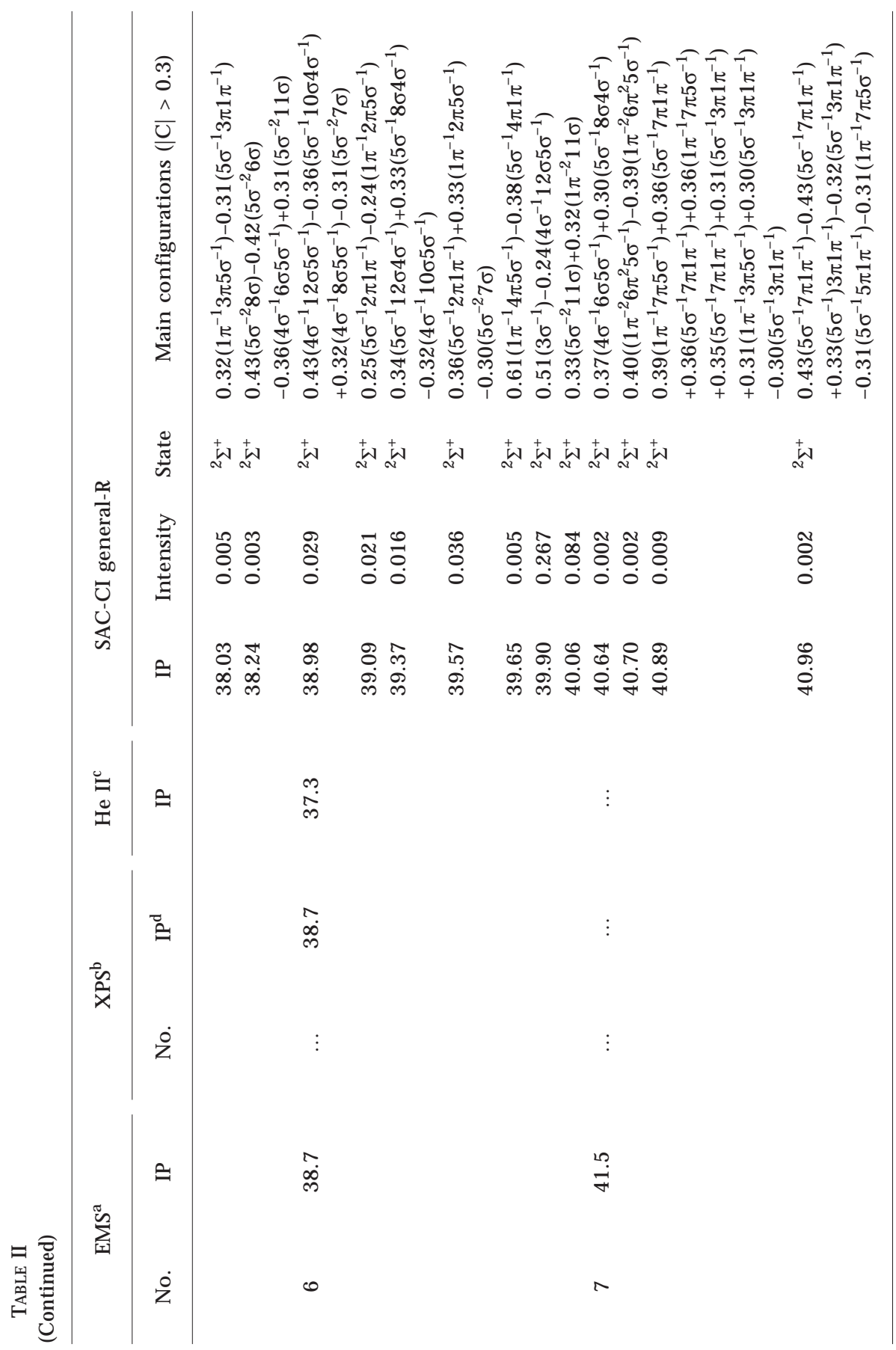




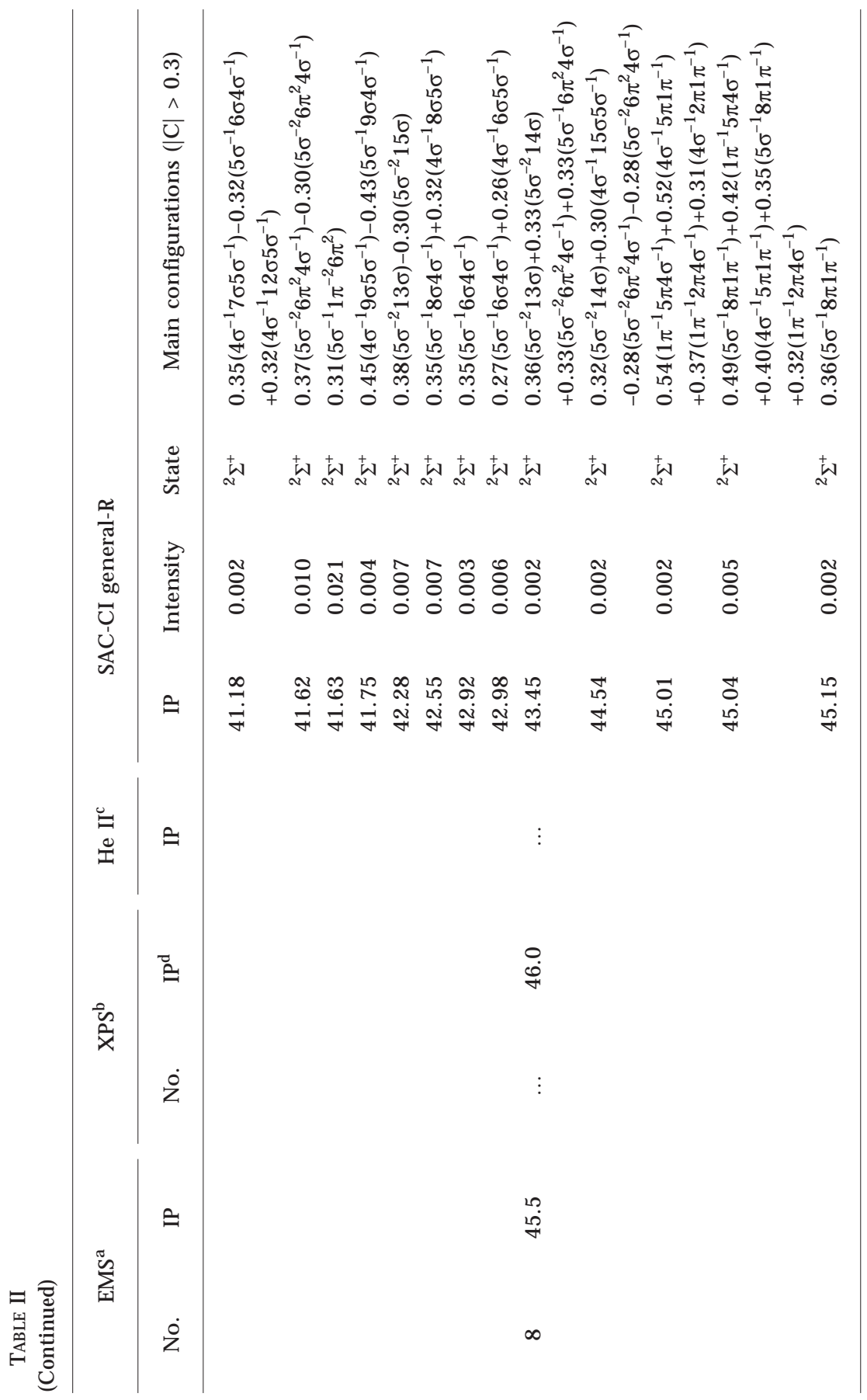




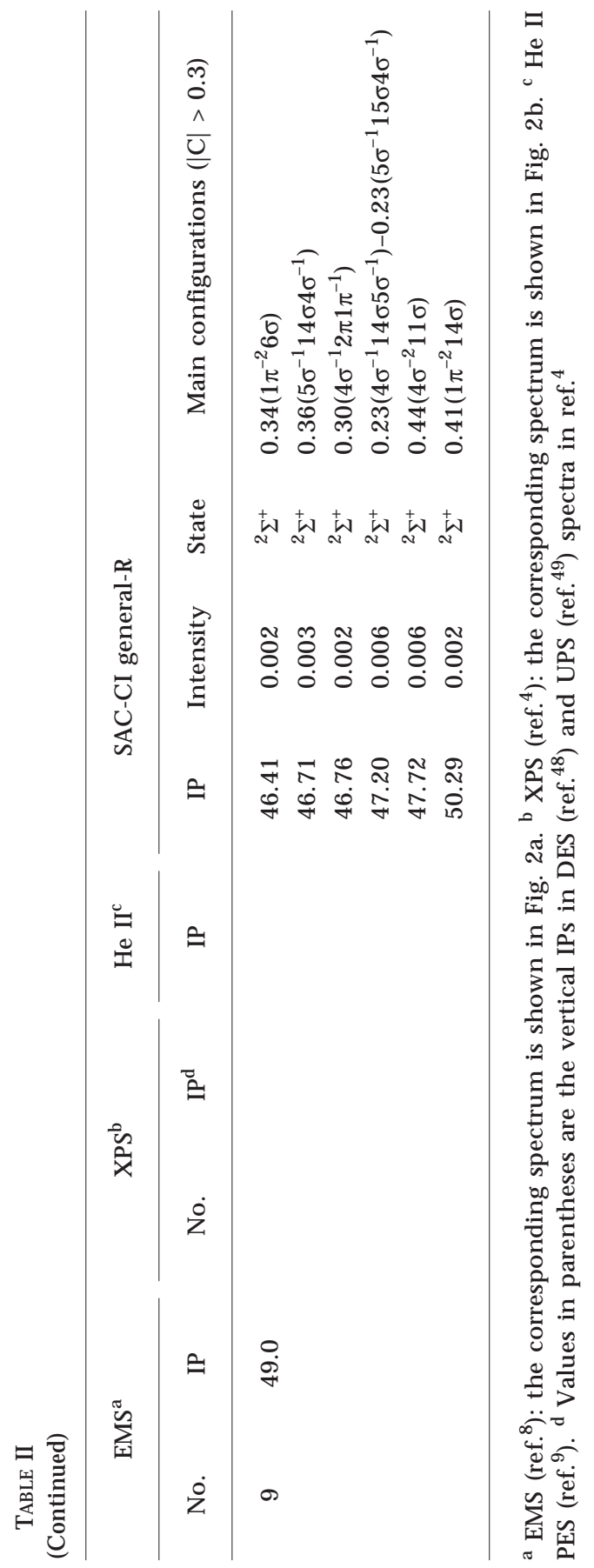



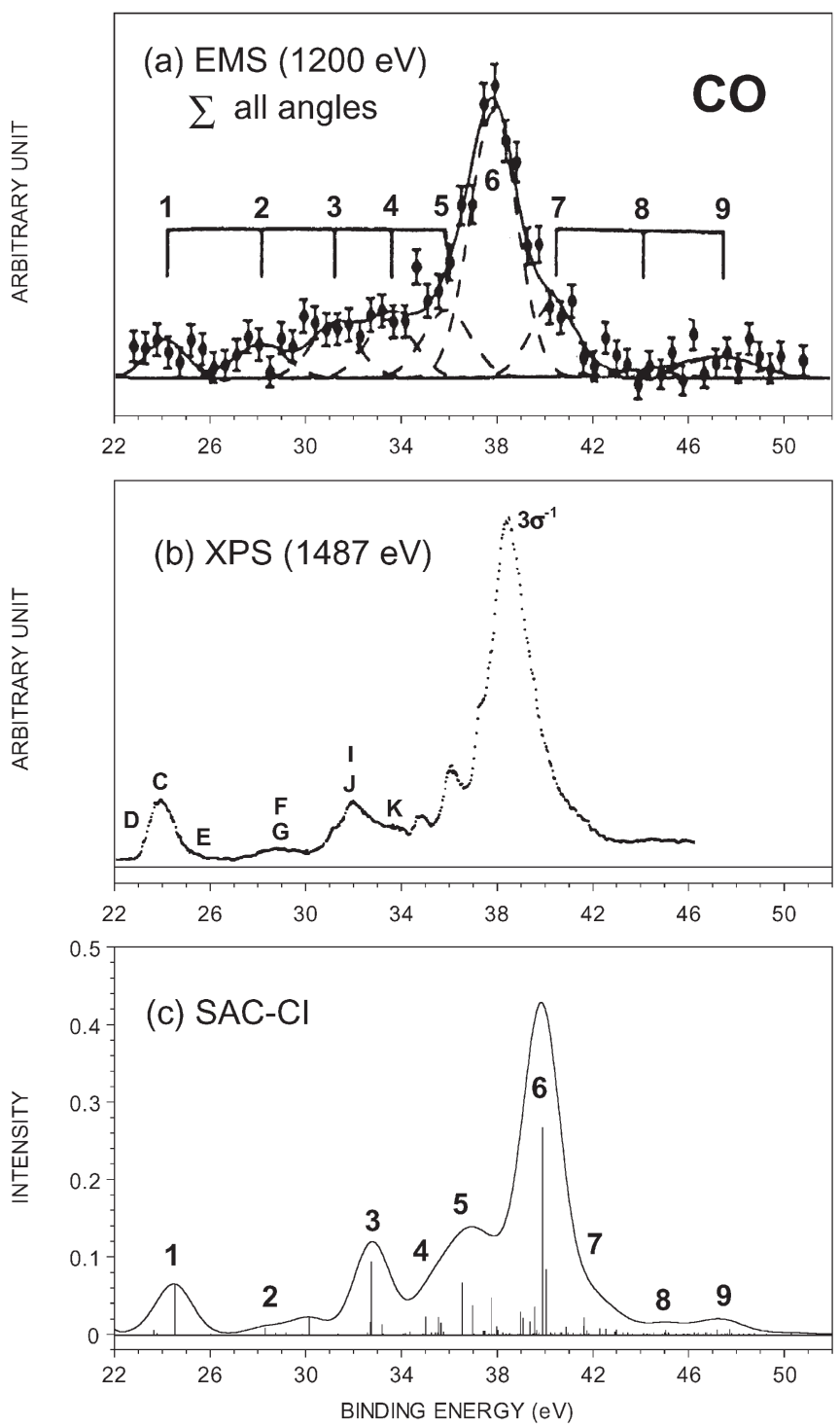

FIG. 2

The inner-valence ionization spectra of CO by a EMS (1200 eV) summed at ten azimuthal angles $^{8}$, b XPS (1487 eV) ${ }^{4}$, and c SAC-CI general-R method. In the theoretical spectrum, the calculated pole strength of each peak is shown as a solid vertical line and is convoluted with a FWHM of $2.5 \mathrm{eV}^{8}$ 
the tiny intensity 0.0003 . Though we assign this ${ }^{2} \Delta$ state to this shoulder, the vibrational progressions of other peaks can overlap in this energy re gion. Therefore, it is necessary to study the vibrational states, the FrankCondon factor, and the intensity dependence on the CO distance for final assignments of the peak $\mathrm{E}$.

Three ${ }^{2} \Pi$ and one ${ }^{2} \Sigma^{+}$states were calculated at 28.31, 28.75, 29.17 and $30.16 \mathrm{eV}$, which were attributed to band 2 observed at $28.3 \mathrm{eV}$ (EMS) ${ }^{8}$ and $28.1 \mathrm{eV}$ (He II PES) ${ }^{9}$. Three ${ }^{2} \Pi$ states at 28.31, 28.75 and $29.17 \mathrm{eV}$ correspond to the $\left(1 \pi^{-1}\right)$ outer-valence satellites and are characterized as the shake-up states including valence-excitations of $\left(4 \sigma^{-1} 5 \sigma^{-1} 6 \pi\right),\left(5 \sigma^{-2} 6 \pi\right)$ and $\left(1 \pi^{-2} 6 \pi\right)$. The total pole strength of these states was calculated to be 0.012 . On the other hand, the ${ }^{2} \Sigma^{+}$state at $30.16 \mathrm{eV}$ with the intensity 0.021 corresponds to the $\left(5 \sigma^{-1}\right)$ outer-valence satellite and is mainly described by $\left(5 \sigma^{-1} 1 \pi^{-1} 6 \pi\right)$. In the XPS spectrum ${ }^{4}$, the corresponding peak appeared as a weak and broad feature centered at about $28.5 \mathrm{eV}$. This peak was interpreted as being due to the two satellites at $27.4 \mathrm{eV}(\mathrm{F})$ and $28.0 \mathrm{eV}(\mathrm{G})$ from the UPS spectrum ${ }^{49}$, and they were assigned to the ${ }^{2} \Pi$ sand ${ }^{2} \Sigma^{+}$states, respectively. In DES ${ }^{48}$, a strong peak was also observed at about $27.6 \mathrm{eV}$. These states were confirmed by theoretical calculations ${ }^{19,51}$, though their assignments were controversial. We assigned three ${ }^{2} \Pi$ states calculated at $28.31,28.75$ and $29.17 \mathrm{eV}$ to the peak $\mathrm{F}$ and the ${ }^{2} \Sigma^{+}$state at $30.16 \mathrm{eV}$ to the peak G, which supports the assignments of the UPS and DES spectra. According to ref. ${ }^{49}$, the peak $G$ forms a vibrational progression centered at about $28.3 \mathrm{eV}$.

Next, three ${ }^{2} \Sigma^{+}$and one ${ }^{2} \Pi$ states were calculated at $32.58,32.74,33.19$ and $32.70 \mathrm{eV}$, which were assigned to band 3 observed at $31.6 \mathrm{eV}(\mathrm{EMS})^{8}$ and $31.8 \mathrm{eV}\left(\mathrm{He}\right.$ II PES) ${ }^{9}$. Three ${ }^{2} \Sigma^{+}$states are due to the $\left(3 \sigma^{-1}\right)$ inner-valence satellites, and are mainly described by $\left(4 \sigma^{-1} 1 \pi^{-1} 6 \pi\right)$ and $\left(5 \sigma^{-2} 11 \sigma\right)$, which involve the valence $\pi^{*}$ excitation for two peaks at 32.58 and $32.74 \mathrm{eV}$, and valence $\sigma^{*}$ excitation for a peak at $33.19 \mathrm{eV}$. The ${ }^{2} \Pi$ state at $32.70 \mathrm{eV}$ is characterized as $\left(1 \pi^{-2} 6 \pi\right)$. In the XPS spectrum, two peaks were discriminated at 31.1 (I) and $32.0 \mathrm{eV}(\mathrm{J})$, and in DES, a weak feature was al so seen at ca. $31 \mathrm{eV}$. We assigned two ${ }^{2} \Sigma^{+}$and one ${ }^{2} \Pi$ states at 32.58. 32.74 and $32.70 \mathrm{eV}$ to the peak I and one ${ }^{2} \Sigma^{+}$state at $33.19 \mathrm{eV}$ to the peak J.

Eight continuous ${ }^{2} \Sigma^{+}$and ${ }^{2} \Pi$ states calculated from 34.36 to $35.76 \mathrm{eV}$ were attributed to the broad band at $34.1 \mathrm{eV}$ (band 4) in EMS. The total pole strength of these states, including peaks with the intensity less than 0.002 , was calculated to be 0.071 . Among these continuous states, three ${ }^{2} \Pi$ states calculated at 34.36, 35.42 and $35.76 \mathrm{eV}$ are mainly described by the two-electron processes including valence $\sigma^{*}$ excitations, such as $\left(5 \sigma^{-1} 1 \pi^{-1} 11 \sigma\right)$ 
and $\left(5 \sigma^{-1} 1 \pi^{-1} 12 \sigma\right)$. Other states correspond to the $\left(3 \sigma^{-1}\right)$ inner-valence satellites: three peaks at 35.25, 35.52 and $35.55 \mathrm{eV}$ are associated with the twoelectron process involving the Rydberg excitations and two peaks at 35.01 and $35.65 \mathrm{eV}$ are due to three-electron processes. Thus, the inclusion of triple and quadruple R-operators is important for describing band 4 . In the XPS spectrum, two peaks were obtained at 33.7 (K) and $34.9 \mathrm{eV}$ in this energy region; we assigned three states from 34.36 to $35.25 \mathrm{eV}$ to the peak $\mathrm{K}$ and other states to the latter peak.

In the energy region above $36 \mathrm{eV}$, the $\left(3 \sigma^{-1}\right)$ inner-valence satellite bands 5-9 were observed by EMS, and these bands lay on the double-ionized states (36-42 eV) 40,53,54. Though there are other possibilities for assignments of these bands, we tentatively assign some shake-up states underlying in the continuum of the double ionized states. Eight ${ }^{2} \Sigma^{+}$states from 36.54 to $38.24 \mathrm{eV}$ were assigned to band 5 observed by EMS at $36.6 \mathrm{eV}$, which is the shoulder of the strongest peak (band 6). The total pole strength of these states, including peaks with small intensity, was calculated to be 0.180 . Among these states, three peaks at $36.98,37.43$ and $37.97 \mathrm{eV}$ are characterized as two- or three-electron processes including $\pi^{*}$ or $\sigma^{*}$ excitations, while others are characterized as the two-electron processes involving the Rydberg excitations. In the XPS spectrum, two peaks were observed at 36.2 and $37.5 \mathrm{eV}$; we assigned two states at 36.54 and $36.98 \mathrm{eV}$ to the former peak and other states from 37.43 to $38.24 \mathrm{eV}$ to the latter peak.

To the strongest band observed by EMS at $38.7 \mathrm{eV}$ (band 6), seven ${ }^{2} \Sigma^{+}$ states calculated from 38.98 to $40.06 \mathrm{eV}$ were attributed. He II PES and XPS observed this peak at 37.3 and $38.7 \mathrm{eV}$, respectively, as the strongest peak in the inner-valence region. The total pole strength of these states, including peaks with small intensity, was calculated to be 0.466 . The prominent two peaks calculated at 39.90 and $40.06 \mathrm{eV}$ with strong intensities are characterized as a linear combination of $\left(3 \sigma^{-1}\right)$ and two-electron processes such as $\left(4 \sigma^{-1} 5 \sigma^{-1} 12 \sigma\right),\left(5 \sigma^{-2} 11 \sigma\right)$ and $\left(1 \pi^{-2} 11 \sigma\right)$, which involve the excitations to $\sigma^{*}$ orbitals.

In the energy region 40.5-50 eV, three weak and broad bands 7-9 were observed by EMS, and only one peak at $46.0 \mathrm{eV}$, which corresponds to band 8 , was recorded by XPS. Numerous states, whose intensities were less than 0.003 , were calculated in this region. Continuous small peaks calculated from 40.5 to $43 \mathrm{eV}$ were assigned to the broad band observed at $41.5 \mathrm{eV}$ (band 7), which is the shoulder of band 6. Most of these states are characterized as the two-electron processes including Rydberg excitations. Three states at $40.70,41.62$ and $41.63 \mathrm{eV}$ are described by the three-electron process involving $\pi^{*}$ excitations. Band 8 observed at $45.5 \mathrm{eV}$ (EMS) and $46.0 \mathrm{eV}$ 
(XPS) was composed of $\approx 40$ shake-up states from 43.5 to $45.5 \mathrm{eV}$ with the total intensity 0.019 , and band 9 at $49.0 \mathrm{eV}$ was composed of $\approx 30$ states from 46 to $52 \mathrm{eV}$ with the total intensity 0.030 . These shake-up states are also due to the two- or three-electron processes including Rydberg excitations.

\section{IONIZATION SPECTRUM OF $\mathbf{N}_{2}$}

Next, the SAC-CI general-R method was applied to the outer- and innervalence ionization spectra of $\mathrm{N}_{2}$ up to about $45 \mathrm{eV}$. Note that some doubleionized states exist from $37.8 \mathrm{eV}^{40}$. Adopting the linear $\mathrm{D}_{2 \mathrm{~h}}$ point group, 40 ionized states were calculated for ${ }^{2} A_{g}$ symmetry, and 20 ionized states for ${ }^{2} \mathrm{~B}_{2 \mathrm{~g}},{ }^{2} \mathrm{~B}_{1 \mathrm{u}}$ and ${ }^{2} \mathrm{~B}_{2 \mathrm{u}}$ symmetries, respectively. The resulting $\mathrm{SAC}-\mathrm{Cl}$ general- $\mathrm{R}$ dimensions were $176571,135907,133293$ and 128863 for ${ }^{2} A_{g}\left({ }^{2} \Sigma_{g}^{+},{ }^{2} \Delta_{g}\right)$, ${ }^{2} \mathrm{~B}_{2 \mathrm{~g}}\left({ }^{2} \Pi_{\mathrm{g}}\right),{ }^{2} \mathrm{~B}_{1 \mathrm{u}}\left({ }^{2} \Sigma_{\mathrm{u}}^{+},{ }^{2} \Delta_{\mathrm{u}}\right)$ and ${ }^{2} \mathrm{~B}_{2 \mathrm{u}}\left({ }^{2} \Pi_{\mathrm{u}}\right)$ symmetries, respectively, as shown in Table I.

The HF electronic configuration of $\mathrm{N}_{2}$ was calculated as (core $)^{4}\left(2 \sigma_{g}\right)^{2}$ $\left(2 \sigma_{u}\right)^{2}\left(3 \sigma_{g}\right)^{2}\left(1 \pi_{u}\right)^{4}$. In Fig. 3, the SAC-CI ionization spectrum of $\mathrm{N}_{2}$ is compared with the SRPES spectrum ${ }^{5}$. In the theoretical spectrum, the line widths of Gaussian convolution are 1.0 and 2.0 eV FWHMs for main and satellite peaks, respectively. Table III summarizes the calculated IPs, monopole intensities and main configurations together with the IPs by four experiments $4,14,48,49$. We presented the ionized states whose intensity was greater than 0.002 . Note that there are still several other outer- and innervalence satellites that are not given in Table III.

Three main peaks due to $\left(3 \sigma_{\mathrm{g}}{ }^{-1}\right),\left(1 \pi_{\mathrm{u}}{ }^{-1}\right)$ and $\left(2 \sigma_{\mathrm{u}}{ }^{-1}\right)$ ionization processes exist in the valence ionization spectrum of $\mathrm{N}_{2}$. The SAC-CI general-R method calculated these main peaks at $15.38,16.91$ and $18.65 \mathrm{eV}$ in good agreement with the experimental IPs by $X^{3} S^{3,4}$ and UPS ${ }^{14}$, as shown in Table III.

The characterization of the inner-val ence spectrum of $\mathrm{N}_{2}$ is still not definitive, although there are extensive experimental ${ }^{3-6,14}$ and theoretical works ${ }^{15,17-21,23-25}$. The earliest study of the inner-valence region of $\mathrm{N}_{2}$ was due to the XPS spectrum reported by Nilsson et al. ${ }^{3}$ Recently, the highresolution XPS spectrum of $\mathrm{N}_{2}$ was observed by Svensson et al. ${ }^{4}$ and the detailed assignments were proposed by comparing with theoretical calculations, UPS ${ }^{49}$ and DES ${ }^{48}$. The fine UPS spectrum was also recorded between 23 and $35 \mathrm{eV}$ with the high-resolution better than $20 \mathrm{meV}^{14}$. We discuss our results comparing with the detailed spectrum by high-resolution XPS 4 and UPS ${ }^{14}$. In Fig. 4, the inner-valence satellite spectrum by the SAC-CI general-R method was compared with two XPS spectra ${ }^{3,4}$. In the theoretical 
spectrum, the calculated pole strengths were convoluted with the FWHM $2.0 \mathrm{eV}$. In the present calculation, eight satellite bands were found by the Gaussian convolution of the peaks, which were numbered 1 to 8 . The spectrum shape of $\mathrm{N}_{2}$ appears to be very similar to that of $\mathrm{CO}$ as a whole, though the intensity of each corresponding band is different, especially for bands 2-5.

In the lower inner-valence region, $a^{2} \Sigma_{u}^{+}$state was calculated at $25.72 \mathrm{eV}$ with the intensity 0.052 , which corresponds to band 1 . This state is the
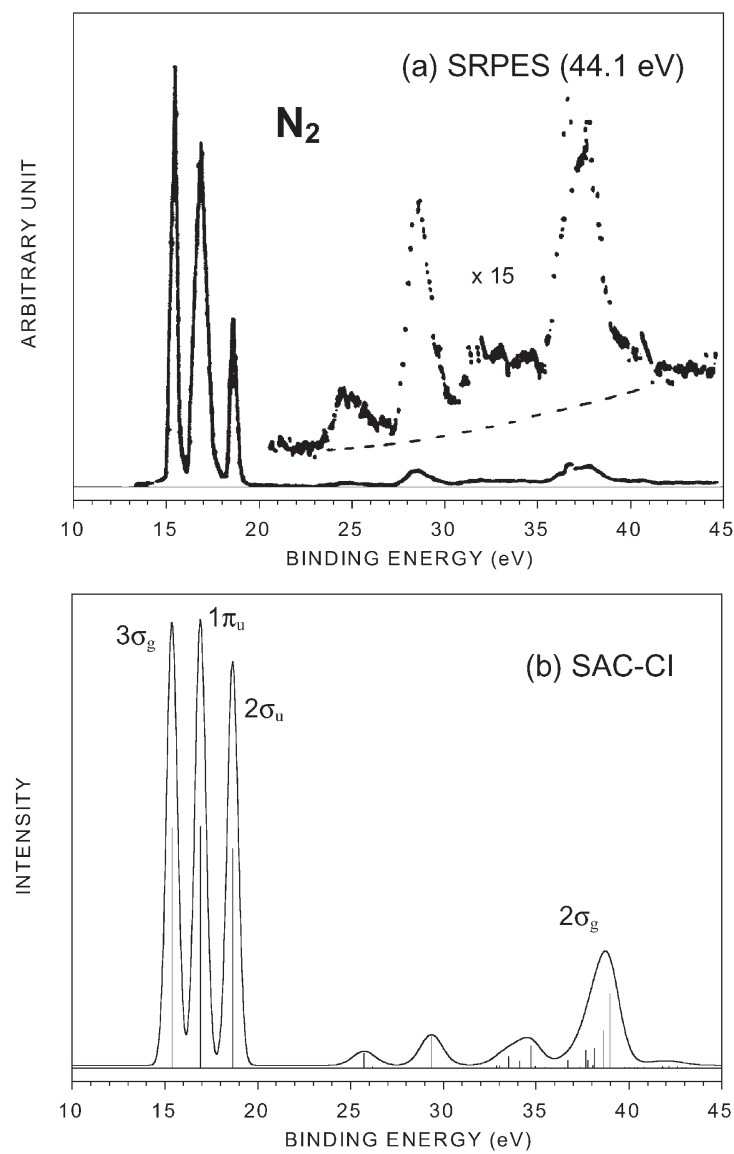

FIG. 3

The valence ionization spectra of $\mathrm{N}_{2}$ by a SRPES $(44.1 \mathrm{eV})^{5}$, and b SAC-Cl general-R method. In the theoretical spectrum, the calculated pole strength of each peak is shown as a solid vertical line and is convoluted with FWHM s of 1.0 and $2.0 \mathrm{eV}$ for main and satellite peaks, respectively 


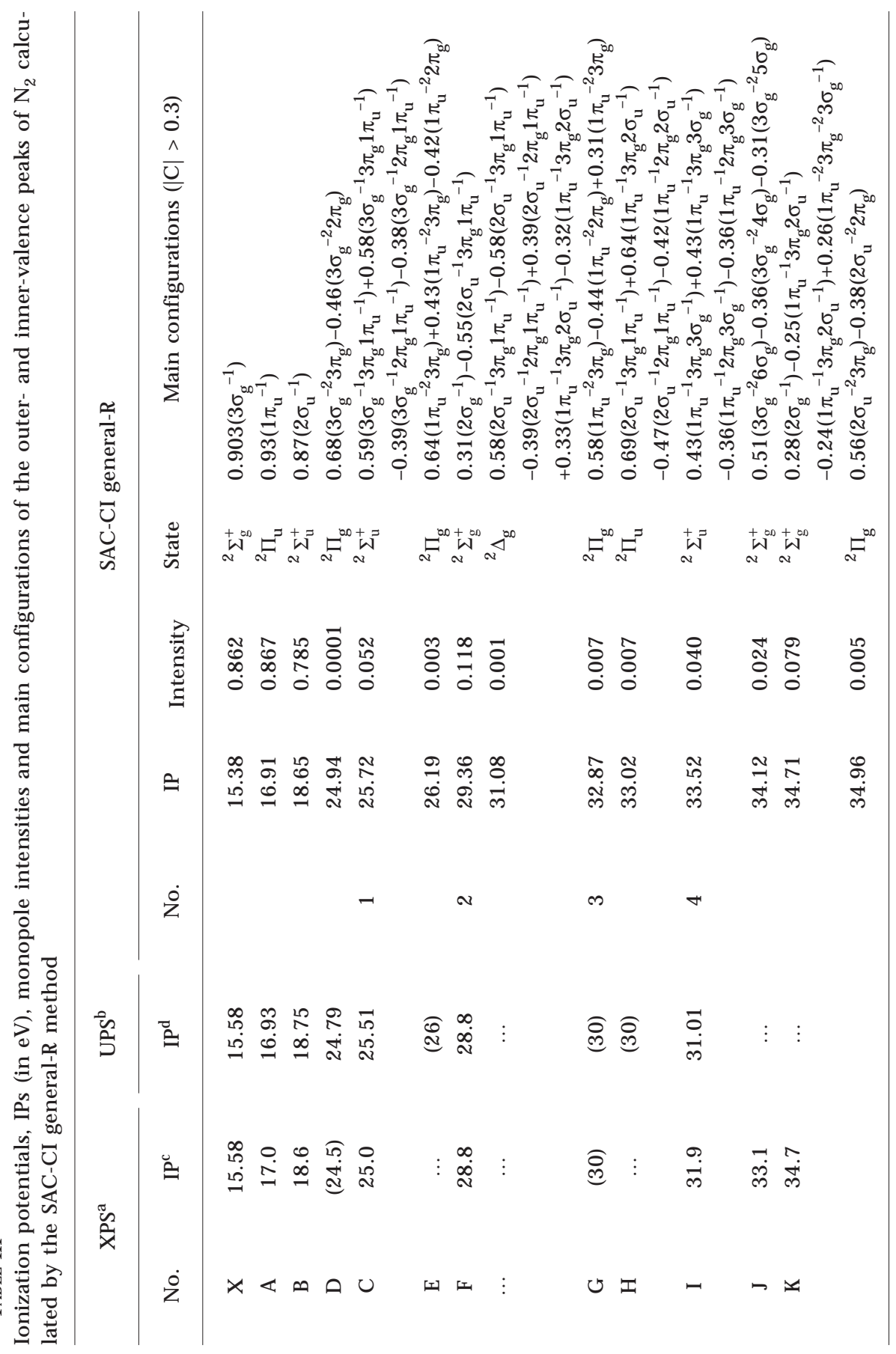




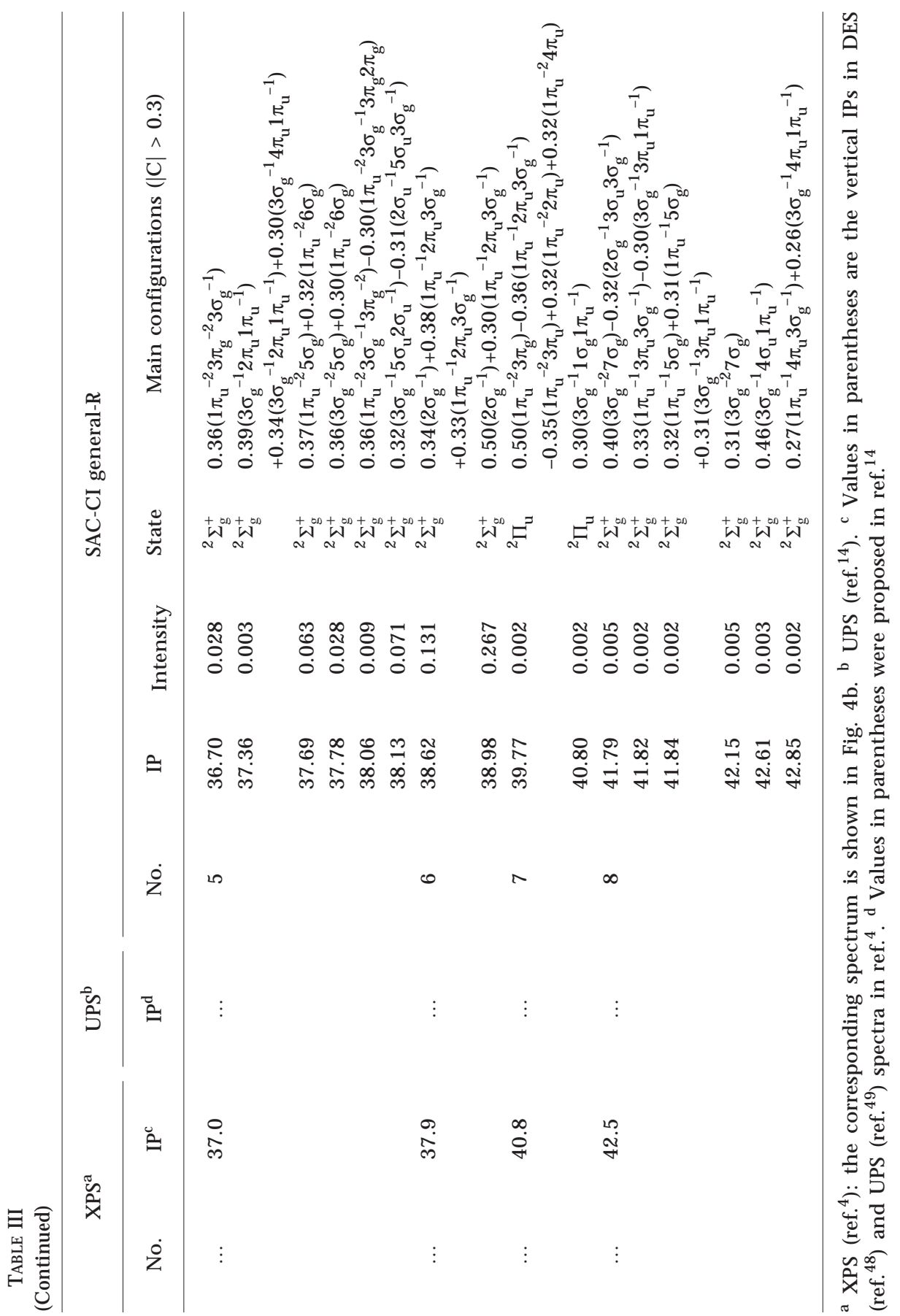



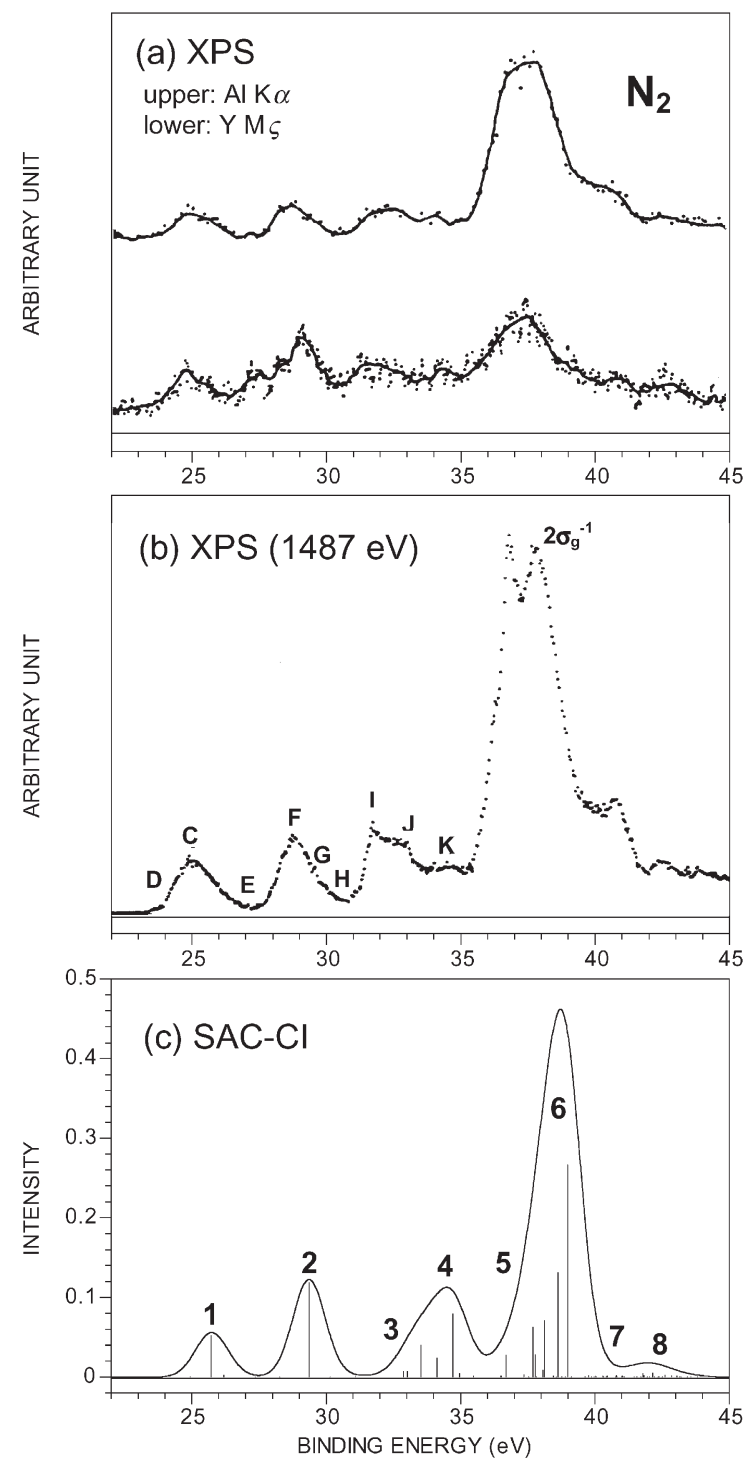

FIG. 4

The inner-valence ionization spectra of $\mathrm{N}_{2}$ by a XPS ${ }^{3}$, b XPS (1487 eV) ${ }^{4}$, and c SAC-Cl general-R method. In the theoretical spectrum, the calculated pole strength of each peak is shown as a solid vertical line and is convoluted with a FWHM of $2.0 \mathrm{eV}$ 
outer-valence satellite of $\left(2 \sigma_{u}^{-1}\right)$ and is described by the two-electron process including valence $\pi^{*}$ excitations, such as $\left(3 \sigma_{\mathrm{g}}{ }^{-1} 3 \pi_{\mathrm{g}} 1 \pi_{\mathrm{u}}{ }^{-1}\right)$. The XPS ${ }^{4}$ and UPS $^{14}$ observed this peak at 25.0 and $25.51 \mathrm{eV}$, respectively, which is denoted as the $C$ state. In the shoulder of band 1 , two shake-up states with ${ }^{2} \Pi$ symmetry have been theoretically predicted as D and E states ${ }^{15,19,21,25}$, which were not reported in the XPS spectra ${ }^{3,4}$. The $C^{2} \Sigma_{\mathrm{u}}^{+}$and $\mathrm{D}^{2} \Pi_{\mathrm{g}}$ states were analyzed in the recent UPS spectrum ${ }^{14}$, in which vibrational progressions of these states were observed with the vertical IPs at 25.514 and $24.788 \mathrm{eV}$, respectively. They also proposed that the vibrational progression of $E^{2} \Pi_{g}$ state could extend up to above $26 \mathrm{eV}$. We assigned two ${ }^{2} \Pi_{\mathrm{g}}$ states calculated at 24.94 and $26.19 \mathrm{eV}$ to the D and E states, respectively, and supported the assignments of the UPS work ${ }^{14}$.

Band 2 is attributed to $a^{2} \Sigma_{\mathrm{g}}^{+}$state calculated at $29.36 \mathrm{eV}$ with the pole strength 0.118 . This state is an inner-valence satellite of $\left(2 \sigma_{\mathrm{g}}{ }^{-1}\right)$ and $\left(2 \sigma_{\mathrm{u}}{ }^{-1} 3 \pi_{\mathrm{g}} 1 \pi_{\mathrm{u}}{ }^{-1}\right)$ including $\pi^{*}$ excitations. This band corresponds to the $\mathrm{F}$ state observed at $28.8 \mathrm{eV}$ in both XPS and UPS spectrum ${ }^{4,14}$. In the energy region above the $F$ state, two shake-up states, ${ }^{2} \Pi_{g}$ and ${ }^{2} \Pi_{u}$, were calculated at 32.87 and $33.02 \mathrm{eV}$, respectively. These states were attributed to band 3 and assigned to $G$ and $H$ states. The $G$ state is characterized as $\left(1 \pi_{u}{ }^{-2} 3 \pi_{g}\right)$ and the $\mathrm{H}$ state as $\left(2 \sigma_{\mathrm{g}}{ }^{-1} 3 \pi_{\mathrm{g}} 1 \pi_{\mathrm{u}}{ }^{-1}\right)$. In the previous experimental ${ }^{5}$ and theoretical ${ }^{19}$ works, two shake-up states were also obtained in this region, but their assignments were different. These states were not observed in the XPS spectra ${ }^{3,4}$. A gren et al. ${ }^{19}$ have calculated two states with ${ }^{2} \Pi_{\mathrm{u}}$ and ${ }^{2} \Sigma_{\mathrm{g}}^{+}$symmetries. In the DES work ${ }^{48}$, the band 2 was observed asymmetrically, which suggested the contribution of the ${ }^{2} \Pi$ state in the high-energy region of this band. The UPS spectrum also observed weak features around $30 \mathrm{eV}$. A weak vibrational progression due to the $G{ }^{2} \Pi_{g}$ state was seen in this energy region; this state was weakly bound according to our calculation ${ }^{50}$. In the recent vibrationally resolved UPS ${ }^{49}$, a weak vibrational progression was also inferred at $30.4 \mathrm{eV}$. We also found the ${ }^{2} \Delta_{\mathrm{g}}$ state at $31.08 \mathrm{eV}$ with quite small intensity in this energy region.

To the peak observed at $31.9 \mathrm{eV}(\mathrm{I})$ by $\mathrm{XPS}^{4}$, we assigned the ${ }^{2} \Sigma_{\mathrm{u}}^{+}$state at $33.52 \mathrm{eV}$ with the intensity 0.04 , which is described by $\left(3 \sigma_{\mathrm{g}}{ }^{-1} 3 \pi_{\mathrm{g}} 1 \pi_{\mathrm{u}}{ }^{-1}\right)$. The IP of this state was overestimated in comparison with experiment. Since this ${ }^{2} \Sigma_{u}^{+}$state is also weakly bound ${ }^{50}$, the vibrational progression can appear in a lower energy region. The UPS experiment also observed a peak at $31.1 \mathrm{eV}^{14}$. Two peaks were observed at 33.1 and $34.7 \mathrm{eV}$ in XPS ${ }^{4}$ corresponding to the peaks $\mathrm{J}$ and $\mathrm{K}$, respectively. The present calculation gave two ${ }^{2} \Sigma_{\mathrm{g}}^{+}$states at 34.12 and $34.71 \mathrm{eV}$ for these peaks. Both of these states 
correspond to $\left(2 \sigma_{g}{ }^{-1}\right)$ inner-valence satellites, though the former state involves the Rydberg excitation the latter being accompanied by the $\pi^{*}$ excitation. In the DES ${ }^{48}$, an increase in the intensity was identified in the energy region 35-36 eV. Accordingly, we calculated a ${ }^{2} \Pi_{\mathrm{g}}$ state at $34.96 \mathrm{eV}$ with the small intensity 0.005 . In this region of peaks I-K, there is discrepancy between the calculated relative intensities and XPS spectra.

The $\left(2 \sigma_{\mathrm{g}}{ }^{-1}\right)$ satellite spectrum was observed in the energy region $36-45 \mathrm{eV} 3,4$. Since numerous states were calculated, one-to-one assignment of the observed peaks would be difficult, however, we proposed our assignment with the shake-up states whose intensity was calculated to be larger than 0.002 . Six ${ }^{2} \Sigma_{g}^{+}$states from 36.70 to $38.13 \mathrm{eV}$ (band 5) were assigned to the shoulder peak at $37.0 \mathrm{eV}$ observed by XPS ${ }^{4}$. The total pole strength of these states was calculated to be 0.204 . Some of these states are mainly characterized as three-electron processes involving the $\pi^{*}$ excitation; the inclusion of quadruple R-operators was important for describing band 5. Two-electron processes involving the Rydberg excitations were also found in this region. The strongest peak was observed at $37.9 \mathrm{eV}$ in the XPS spectrum ${ }^{4}$. We assigned two ${ }^{2} \Sigma_{g}^{+}$states calculated at 38.62 and $38.98 \mathrm{eV}$ (band 6), which are described by $\left(2 \sigma_{\mathrm{g}}{ }^{-1}\right)$ and $\left(1 \pi_{\mathrm{u}}{ }^{-1} 2 \pi_{\mathrm{u}} 3 \sigma_{\mathrm{g}}{ }^{-1}\right)$ including the Rydberg excitations. The total pole strength of these states was calculated to be $\approx 0.4$.

Numerous ${ }^{2} \Sigma_{g}^{+},{ }^{2} \Pi_{g},{ }^{2} \Sigma_{u}^{+}$and ${ }^{2} \Pi_{u}$ states were calculated from 39 to $41.4 \mathrm{eV}$ (band 7) and from 41.5 to $45 \mathrm{eV}$ (band 8) in the higher-energy region of band 6 . The former band 7 was attributed to the shoulder observed at $40.8 \mathrm{eV}$ in the XPS ${ }^{4}$. In the DES spectrum ${ }^{52}$, weak features were observed around $40 \mathrm{eV}$; we calculated two ${ }^{2} \Pi_{\mathrm{u}}$ outer-valence satellites at 39.77 and $40.8 \mathrm{eV}$ with the intensity 0.002 . Band 8 was attributed to a weak and broad band observed at $42.5 \mathrm{eV}$ by XPS ${ }^{4}$, which is mainly described as the two-electron processes involving the Rydberg excitations.

\section{CONCLUSIONS}

The outer- and inner-valence ionization spectra of $\mathrm{CO}$ and $\mathrm{N}_{2}$ were studied using the $\mathrm{SAC}-\mathrm{Cl}$ general-R method with flexible basis sets. The SAC-Cl general-R method quite accurately reproduced the experimental spectra of these molecules and gave the detailed assignment of the satellite peaks.

In both the outer- and inner-valence region, satel lite peaks were classified into the ionization including valence or Rydberg excitations. Our results show that numerous shake-up states exist with distributed intensities, especially in the high-energy region, and they constitute several bands that were not experimentally resolved. This picture was less clear in the previous 
studies. In particular, we calculated the detailed spectrum of the shake-up states including Rydberg excitations, for which the Rydberg functions should be included and numerous states should be solved in the calculations.

For CO, we presented the theoretical satellite spectrum up to $50 \mathrm{eV}$, which interpreted the nine bands observed by EMS in 20-50 eV and the eight bands by XPS in 20-34 eV. The first band was assigned to ${ }^{2} \Sigma^{+}$and ${ }^{2} \Pi$ states for peaks $C$ and $D$, respectively. For peak $E$, we calculated no states with ${ }^{2} \Sigma^{+}$or ${ }^{2} \Pi$ symmetries, but a ${ }^{2} \Delta$ state with tiny intensity. For this peak, vibrational progressions of other peaks can al so contribute. We assigned the ${ }^{2} \Pi$ and ${ }^{2} \Sigma^{+}$states for peaks $F$ and $G$, respectively, whose assignment has not been definitive yet. For these peaks, our assignments are consistent with the DES and UPS spectra ${ }^{48,49}$. We calculated ${ }^{2} \Sigma^{+}$satellite states corresponding to peaks I and J. Numerous ${ }^{2} \Sigma^{+}$and ${ }^{2} \Pi$ satellite peaks were obtained in the higher energy region 34-52 eV and some of them, especially for bands 4, 5, 6 and 7, were predominantly described by triple-electron processes; the inclusion of quadruple R-operators was important for describing these states in the SAC-CI general-R method.

For $\mathrm{N}_{2}$, we calculated the spectrum up to $45 \mathrm{eV}$ and interpreted the complicated satellite peaks (C-K) in the energy region 20-35 eV. We assigned two ${ }^{2} \Pi_{\mathrm{g}}$ states to the $D$ and $E$ states and supported the assignments of UPS spectrum ${ }^{14}$. We calculated three outer-valence satellites for the $\mathrm{G}, \mathrm{H}$ and I states, which were reported by recent high-resolution XPS ${ }^{4}$. More detailed analysis of the vibrational states would be necessary for final assignment, especially for peaks I, J and $\mathrm{K}$. In the higher-energy region 33-45 eV, the detailed assignments for the $\left(2 \sigma_{\mathrm{g}}{ }^{-1}\right)$ inner-valence satellites were given. We found that $\left(2 \pi_{\mathrm{u}}{ }^{-1}\right)$ outer-val ence satellite states exist in band 7 in agreement with the DES spectrum ${ }^{52}$.

Finally, it is further necessary to investigate the vibrational spectrum of the satellite peaks for complete assignments, especially for the lower energy region. Theoretical study on vibrational levels of the satellites using the $\mathrm{SAC}-\mathrm{Cl}$ general-R method is now in progress ${ }^{50}$.

This study has been supported by the Grant for Creative Scientific Research from the Ministry of Education, Science, Culture, and Sports of Japan.

\section{REFERENCES}

1. Siegbahn K., Nordling C., Johannson G., Hedman J., Heden P. F., Hamrin K., Gelius U., Bergmark T., Werme L. O., Manne R., Baer Y.: ESCA Applied to Free Molecules. NorthHolland, Amsterdam 1969. 
2. Gelius U., Basilier E., Svensson S., Bergmark T., Siegbahn K.: J. Electron Spectrosc. Relat. Phenom. 1973, 2, 405.

3. Nilsson R., Nyholm R., Berndtsson A., Hedman J., Nordling C.: J. Electron Spectrosc. Relat. Phenom. 1976, 9, 337.

4. Svensson S., Carlsson-Göthe M., Karlsson L., Nilsson A., Mårtensson N., Gelius U.: Phys. Scr. 1991, 44, 184.

5. Krummacher S., Schmit V., Wuilleumier F.: J. Phys. B: At., Mol. Opt. Phys. 1980, 13, 3993.

6. Krummacher S., Schmit V., Wuilleumier F., Bizau J. M., Ederer D.: J. Phys. B: At., Mol. Opt. Phys. 1983, 16, 1733.

7. Dey S., Dixon A. J., Lassey K. R., McCarthy I. E., Teubner P. J. O., Weigold E., Bagus P. S., Viinikka E. K.: Phys. Rev. A: At., Mol., Opt. Phys. 1977, 15, 102.

8. French C. L., Brion C. E., Bawagan A. O., Bagus P. S., Davidson E. R.: Chem. Phys. 1988, $121,315$.

9. Potts A. W., Williams T. A.: J. Electron Spectrosc. Relat. Phenom. 1974, 3, 3.

10. Åsbrink L., Fridh C., Lindholm E., Codling K.: Phys. Scr. 1974, 10, 183.

11. Allison D. A., Gavell R. G.: J. Chem. Phys. 1978, 68, 593.

12. Liu Z. F., Bancroft G. M., Coatsworth L. L., Tan K. H.: Chem. Phys. Lett. 1993, 203, 337.

13. Baltzer P., Lundqvist M., Wannberg B., Karlsson L., Larsson M., Hayes M. A., West J. B., Siggel M. R. F., Parr A. C., Dehmer J. L.: J. Phys. B: At., Mol. Opt. Phys. 1994, 27, 4915.

14. Baltzer P., Larsson M., Karlsson L., Wannberg B., Carlsson-Göthe M.: Phys. Rev. A: At., Mol., Opt. Phys. 1992, 46, 5545.

15. Okuda M., Jonathan N.: J. Electron Spectrosc. Relat. Phenom. 1974, 3, 19.

16. Bagus P. S., Viinikka E. K.: Phys. Rev. A: At., Mol., Opt. Phys. 1977, 15, 1486.

17. Kosugi N., Kuroda H., Iwata S.: Chem. Phys. 1979, 39, 337.

18. Honjou N., Sasajima T., Sasaki F.: Chem. Phys. 1981, 57, 475.

19. Ågren H., Arneberg R., Müller J., Manne R.: Chem. Phys. 1984, 83, 53.

20. Langhoff P. W., Langhoff S. R., Rescigno T. N., Schirmer J., Cederbaum L. S., Domcke W., von Niessen W.: Chem. Phys. 1981, 58, 71.

21. Schirmer J., Cederbaum L. S., Domcke W., von Niessen W.: Chem. Phys. 1977, 26, 149.

22. von Niessen W., Cederbaum L. S., Schirmer J., Diercksen G. H. F., Kraemer W. P.: J. Electron Spectrosc. Relat. Phenom. 1982, 28, 45.

23. Schirmer J., Walter O.: Chem. Phys. 1983, 78, 201.

24. Ehara M., Nakatsuji H.: Chem. Phys. Lett. 1998, 282, 347.

25. Nichols J. A., Yeager D. L., Jörgensen P.: J. Chem. Phys. 1984, 80, 293.

26. Turner D. W., Baker C., Baker A. D., Brundle C. R.: Molecular Photoelectron Spectroscopy. Wiley, New York 1970.

27. Nakatsuji H., Hirao K.: J. Chem. Phys. 1978, 68, 2053.

28. a) Nakatsuji H.: Chem. Phys. Lett. 1978, 59, 362; b) Nakatsuji H.: Chem. Phys. Lett. 1979, 67, 329; c) Nakatsuji H.: Chem. Phys. Lett. 1979, 67, 334; d) Nakatsuji H.: Acta Chim. Hung. Models Chem. 1992, 129, 719; e) Nakatsuji H.: Computational Chemistry Reviews of Current Trends, Vol. 2, p. 62. World Scientific, Singapore 1997.

29. Nakatsuji H., Hirao K.: Chem. Phys. Lett. 1981, 79, 299.

30. Nakatsuji H.: Chem. Phys. 1983, 75, 425.

31. Nakatsuji H., Kitao O., Yonezawa T.: J. Chem. Phys. 1985, 83, 723.

32. a) Nakatsuji H., Saito S.: J. Chem. Phys. 1989, 91, 6205; b) Nakatsuji H., Ehara M., Palmer M. H., Guest M. F.: J. Chem. Phys. 1992, 97, 2561; c) Nakatsuji H., Ehara M.: J. Chem. Phys. 1994, 101, 7658; d) Nakatsuji H., Hasegawa J., Hada M.: J. Chem. Phys. 1996, 104, 2321. 
33. Ehara M., Ohtsuka Y., Nakatsuji H.: Chem. Phys. 1998, 226, 113.

34. Ehara M., Nakatsuji H.: Spectrochim. Acta, Part A 1999, 55, 487.

35. Hasegawa J., Ehara M., Nakatsuji H.: Chem. Phys. 1998, 230, 23.

36. Ehara M., Tomasello P., Hasegawa J., Nakatsuji H.: Theor. Chem. Acc. 1999, 102, 161.

37. a) Ehara M., Ishida M., Nakatsuji H.: J. Chem. Phys. 2001, 114, 8990; b) Ishida M., Ehara M., Nakatsuji H.: J. Chem. Phys. 2002, 116, 1934.

38. a) Nakatsuji H.: Chem. Phys. Lett. 1991, 177, 331; b) Nakatsuji H.: J. Chem. Phys. 1985 , 83, 713; c) Nakatsuji H.: J. Chem. Phys. 1985, 83, 5743; d) Nakatsuji H.: J. Chem. Phys. 1991, 94, 6716 .

39. Ehara M., Ishida M., Toyota K., Nakatsuji H. in: Reviews in Modern Quantum Chemistry (K. D. Sen, Ed.), p. 293. World Scientific, Singapore 2002.

40. Huber K. P., Herzberg G.: Molecular Spectra and Molecular Structure, IV. Constants of Diatomic Molecules. Van Nostrand, New York 1979.

41. Schafer A., Horn H., Ahlrichs R.: J. Chem. Phys. 1992, 97, 2571.

42. a) Dunning T. H., Jr.: J. Chem. Phys. 1989, 90, 1007; b) Woon D. E., Dunning T. H., Jr.: J. Chem. Phys. 1993, 98, 1358.

43. Dunning T. H., Hay P. J.: Methods of Electronic Structure Theory (H. F. Schaefer III, Ed.), Vol. 3. Plenum Press, New York 1977.

44. Süzer S., Lee S. T., Shirley D. A.: Phys. Rev. A: At., Mol., Opt. Phys. 1976, 13, 1842.

45. Martin R. I., Shirley D. A.: J. Chem. Phys. 1976, 64, 3685.

46. Frisch M. J., Trucks G. W., Schlegel H. B., Scuseria G. E., Robb M. A., Cheeseman J. R., Zakrzewski V. G., Montgomery J. A., Jr., Stratmann R. E., Burant J. C., Dapprich S., Millam J. M., Daniels A. D., Kudin K. N., Strain M. C., Farkas O., Tomasi J., Barone V., Cossi M., Cammi R., Mennucci B., Pomelli C., Adamo C., Clifford S., Ochterski J., Petersson G. A., Ayala P. Y., Cui Q., Morokuma K., Malick D. K., Rabuck A. D., Raghavachari K., Foresman J. B., Cioslowski J., Ortiz J. V., Stefanov B. B., Liu G., Liashenko A., Piskorz P., Komaromi I., Gomperts R., Martin R. L., Fox D. J., Keith T., Al-Laham M. A., Peng C. Y., Nanayakkara A., Gonzales C., Challacombe M., Gill P. M. W., Johnson B., Chen W., Wong M. W., Andres J. L., Gonzales C., Head-Gordon M., Replogle E. S., Pople J. A.: Gaussian 98, Revision A.5. Gaussian, Inc., Pittsburgh (PA) 1998.

47. Nakatsuji H., Hada M., Ehara M., Hasegawa J., Nakajima T., Nakai H., Kitao O., Toyota K.: SAC/SAC-CI Program System (SAC-CI96) for calculating ground, excited, ionized, and electron-attached states having singlet to septet spin multiplicities. Computer Center of the Institute for Molecular Science, Okazaki 1996.

48. Eberhardt W., Plummer E. W., Lyo I.-W., Murphy R., Carr R., Ford W. K.: J. Phys. (Paris) Colloque C9, Suppl. 1987, 12, 697.

49. Baltzer P., Carlsson-Göthe M., Wannberg B., Karlsson L., Svensson S.: Uppsala Univ. Inst. Phys. Rep. 1990, UUIP-1233.

50. Ehara M., Ishida M., Toyota K., Nakatsuji H.: Unpublished results.

51. Freund H. J., Llegener C. M.: Chem. Phys. Lett. 1987, 134, 70.

52. Eberhardt W., Plummer E. W., Chen C. T., Ford D. K.: Aust. J. Phys. 1986, 39, 853.

53. Hochlaf M., Hall R. I., Penent F., Kjeldsen H., Lablanquie P., Lavollee M., Eland J. D. H.: Chem. Phys. 1996, 207, 159.

54. Thompson D. B., Dawber G., Gulley N., MacDonald M. A., King G. C.: J. Phys. B: At., Mol. Opt. Phys. 1997, 30, L147. 